\title{
Nonalcoholic Fatty Liver Disease in Children
}

\author{
Jake P. Mann, MBChB ${ }^{1}$ Luca Valenti, MD ${ }^{2}$ Eleonora Scorletti, $\mathrm{MD}^{3}$ \\ Christopher D. Byrne, MBChB, PhD ${ }^{3,4,5}$ Valerio Nobili, MD ${ }^{6,7}$
}

${ }^{1}$ Department of Paediatrics, University of Cambridge, Cambridge, United Kingdom

${ }^{2}$ Department of Pathophysiology and Transplantation, Fondazione IRCCS Ca' Granda Ospedale Policlinico Milano, Università degli Studi di Milano, Milan, Italy

${ }^{3}$ Human Development and Health Academic Unit, Faculty of Medicine, University of Southampton, Southampton, United Kingdom

${ }^{4}$ National Institute for Health Research Southampton Biomedical Research Centre (in Nutrition), University of Southampton and University Hospital Southampton National Health Service (NHS) Foundation Trust, Southampton, United Kingdom

${ }^{5}$ Respiratory Biomedical Research Unit, University of Southampton and University Hospital Southampton National Health Service (NHS) Foundation Trust, Southampton, United Kingdom

${ }^{6}$ Hepatometabolic Disease Unit, Bambino Gesù Children's Hospital, IRCCS (Instituto di Ricovero e Cura a Carattere Scientifico), Rome, Italy

7 Department of Pediatrics, University “La Sapienza," Rome, Italy

Address for correspondence Valerio Nobili, MD, Department of Pediatrics, University “La Sapienza," and Hepatometabolic Disease Unit, Bambino Gesù Children's Hospital IRCCS (Instituto di Ricovero e Cura a Carattere Scientifico), P.le S. Onofrio 4, 00165 Rome, Italy (e-mail: nobili66@yahoo.it).

Semin Liver Dis 2018;38:1-13.

\begin{abstract}
Nonalcoholic steatohepatitis, a progressive form of nonalcoholic fatty liver disease (NAFLD), is one of the most common hepatic diseases in children who present with particular risk factors including obesity, sedentary lifestyle, and/or a predisposing genetic background. The worldwide prevalence of NAFLD in children is a worrying phenomenon because this disease is closely associated with the development of both cirrhosis and cardiometabolic syndrome in adulthood. To date, the etiopathogenesis of primary NAFLD in children is unknown. Understanding the pathogenetic mechanisms provides the basis to characterize early predictors of the disease and noninvasive diagnostic tools and to design

Keywords

- NAFLD

- obesity

- children

- NASH novel specific treatments and possible management strategies. Despite a few clinical trials on the use of antioxidants combined with lifestyle intervention for NAFLD, no treatment exists for children with NAFLD. In this review, the authors provide an overview of current concepts in epidemiology, histological features, etiopathogenesis, diagnosis, and treatment of NAFLD in pediatric population.
\end{abstract}

Nonalcoholic fatty liver disease (NAFLD) is a multifactorial disorder closely associated with the metabolic syndrome and is the most common cause of abnormal liver function tests (LFTs) in children. NAFLD is set to become the major cause of liver transplantation in adults, and while it is rare for children to develop end-stage liver disease from NAFLD, they may become cirrhotic as adults. Understanding and managing NAFLD in children may represent a method to intervene early and alter the disease process. Furthermore, children with steatosis require careful assessment as it may be secondary to other conditions (e.g., Wilson's disease [WD]). For these reasons, pediatric NAFLD is of high importance to all gastroenterologists, hepatologists, and pediatricians.

NAFLD refers to a spectrum of diseases, ranging from hepatic steatosis ("simple steatosis" or nonalcoholic fatty liver [NAFL]) to nonalcoholic steatohepatitis (NASH) with or without fibrosis, to end-stage liver disease. Diagnosis requires radiological (or histological) demonstration of steatosis, exclusion of secondary causes, and no significant alcohol intake. Children present in three main ways: incidental abnormal LFT/
Copyright $\odot 2018$ by Thieme Medical Publishers, Inc., 333 Seventh Avenue, New York, NY 10001, USA. Tel: +1(212) 584-4662. 
imaging, screening in obesity, and during investigation of abdominal pain, the cause of which is not clear.

In this review, we aim to provide an overview of pediatric NAFLD and discuss management in the context of recent guidance.

\section{Epidemiology}

Paralleling the dramatic rise in pediatric obesity worldwide, NAFLD has become a leading cause of chronic liver disease during the developmental age, and the main determinant on pediatric liver disease in Western countries. ${ }^{1}$ Several studies have demonstrated a prevalence of 3 to $10 \%$ in general pediatric populations, which increases up to 60 to $70 \%$ in individuals with metabolic comorbidities. ${ }^{2}$ However, NAFLD prevalence varies widely depending on geographical area and diagnostic methods used.

Initial population-based studies, which estimated the prevalence of pediatric NAFLD by determining aminotransferases or by ultrasonography in several countries, have indicated a prevalence range of 3 to $7 \%{ }^{1}$ In an autoptic study, conducted in unselected children who died in accidents in California, the prevalence of histological NAFLD ranged from $0.7 \%$ in 2 - to 4-year-old to $17.3 \%$ in 15- to 19 -year-old subjects, but increased to $38 \%$ in obese children. ${ }^{3}$ In cohorts of children of various nationalities selected for overweight or obesity, the prevalence of elevated alanine aminotransferase (ALT) was higher and ranged from 8 to $42 \%$, whereas the prevalence of bright liver ranged from 1.7 to $77 \%{ }^{4} \mathrm{~A}$ recent attempt to examine NAFLD prevalence based on a meta-analysis of studies conducted in 76 different populations led to an estimate of 7.6\% (95\% confidence interval $[\mathrm{CI}]: 5.5-10.3 \%$ ) in the general population and $34.2 \%$ (95\% CI: $27.8-41.2 \%$ ) in obesity clinics. ${ }^{5}$ However, there was a huge heterogeneity $\left(I^{2}=98 \%\right)$, which was partly accounted for by sex distribution, difference in body mass index (BMI), and ethnicity, with NAFLD prevalence being higher in males, individuals with more severe adiposity, and Asians. Importantly, use of liver enzymes instead of imaging to diagnose NAFLD led to a significant underestimation of disease prevalence. $^{5}$

Indeed, obesity and metabolic syndrome features are the major risk factors for pediatric NAFLD. The prevalence of this condition is higher in overweight (gender- and age-specific BMI $>85$ th percentile) or obese ( $>95$ th) children as compared with normal weight pairs. However, due to the closest link to insulin resistance, central adiposity, which is accumulation of fat in visceral organs, plays a specific contribution in determining disease risk. ${ }^{6,7}$ Indeed, waist circumference, an easily available index of visceral fat, correlates with NAFLD independently of BMI. ${ }^{1,8}$

Nutritional factors, such as excessive intake of calories, processed food, and a sedentary lifestyle, play a key role in the predisposition to liver fat accumulation, NAFLD, and progressive liver disease. ${ }^{9,10}$ Fructose intake has emerged as an important determinant of NAFLD risk, independently of total caloric intake, probably due to the ability to stimulate de novo lipogenesis (DNL). ${ }^{11,12}$ This has recently been demonstrated to translate into increased risk of $\mathrm{NASH}^{13}$
The quality of fat, and specifically a reduced omega- 3 : omega-6 ratio, has also been reported to predispose to NAFLD in children at higher risk. ${ }^{14}$ On the other hand, high levels of physical activity are associated with protection from NAFLD. ${ }^{8}$

Inherited factors account for a large proportion of the interethnic and interindividual variability in the predisposition to NAFLD. Genetic studies have now identified the specific common variants that influence hepatic fat metabolism as important determinants of NAFLD in children and adults. ${ }^{15-17}$ Also, in the developmental age, NAFLD is more prevalent in individuals of Hispanic and Asian ethnicities as compared with Europeans, whereas those of African ancestry are relatively protected. ${ }^{5,18}$ The most validated genetic risk factors are the PNPLA3 I148M and TM6SF2 E167K mutations that influence lipid droplet remodeling and the secretion of lipids from hepatocytes, ${ }^{19-21}$ GCKR P446L regulating lipogenesis, ${ }^{22}$ and genetic variation in the MBOAT7 gene that affects acyl chain remodeling of phosphatidylinositol. ${ }^{17}$ Evaluation of these genetic risk variants increases the ability to stratify the risk of NAFLD. ${ }^{23}$ Recent studies also suggest a link between NAFLD and epigenetic modifications and stable changes in expression of DNA related to chemical modifications of DNA and chromatin structure, caused by exposure to environmental factors. ${ }^{24}$ In fact, accumulating evidence suggests that an adverse intrauterine environment as detected by low birth weight is associated with increased risk of pediatric and adult NAFLD. ${ }^{23,25}$

\section{Histology}

From a histopathological point of view, NAFLD encompasses a disease spectrum ranging from "simple steatosis" (NAFL) to $\mathrm{NASH}$, which is characterized by the presence of hepatocellular damage under the form of ballooning and mixed lobular inflammation, is associated with the activation of pericellular-perisinusoidal fibrogenesis, and evolves to fibrosis, cirrhosis, and hepatocellular carcinoma (HCC) in a variable proportion of cases. ${ }^{26}$ Other common features include Mallory-Denk bodies, megamitochondria, acidophil bodies, and iron accumulation. ${ }^{27}$

NASH is associated with faster progression of liver fibrosis as compared with simple steatosis. ${ }^{28}$ Importantly, fibrosis stage is the main determinant of the prognosis of patients with NAFLD, concerning both liver-related and overall mortality. ${ }^{29-32}$

Pediatric NAFLD displays some peculiar histological features compared with the adult form and, unlike the adult disease, is rarely influenced by some secondary lifestyle factors (e.g., alcohol and drugs), whereas others may have a more prominent role (e.g., fructose intake). Indeed, two different types of histological damage have been described in children with NAFLD: ${ }^{33}$ "type 1" NAFLD and "type 2" NAFLD. Type 1 NAFLD refers to the histological features classically associated with NASH in obese adults. These are represented by steatosis, that is, accumulation of neutral lipids within intracellular lipid droplets, which is generally more severe in the centrilobular area. This is accompanied by hepatocellular damage in the form of ballooning, lobular inflammation, and/ 
or perisinusoidal fibrosis. In contrast, type 2 NAFLD has been described more frequently during the developmental age and is characterized by steatosis with portal inflammation and/or periportal fibrosis. This form is more commonly observed in males and in children of Hispanic or Asian ethnicity as compared with Europeans. ${ }^{33}$ However, in the majority of series, it was found that although type 2 features are more frequent, most pediatric patients display overlapping features of type 1 and type 2 NAFLD, which can be considered the two extremes of a pathological spectrum. ${ }^{34-36}$ Importantly, portal inflammation, which is typical of type 2 NAFLD, has been associated with more severe fibrosis stage. ${ }^{37}$

In a recent series of 440 Caucasian children with histological NAFLD, $12 \%$ had type $1,22 \%$ had type 2 , and $66 \%$ had overlapping features. ${ }^{38}$ Remarkably, those with type 2 had a more severe metabolic phenotype, with higher central adiposity and dyslipidemia. Furthermore, the presence of portal inflammation, a feature of type 2 NAFLD, was independently associated with waist circumference and clinically significant fibrosis, suggesting that this histological feature is involved in mediating faster progression of the disease.

\section{Pathogenesis of Nonalcoholic Fatty Liver Disease in Children}

The pathogenesis of NAFLD in adults has been welldefined. ${ }^{39-42}$ In contrast, the pathogenesis of NAFLD in children has not been the object of comparable attention, and thus its contributing factors have yet to be mapped comprehensively and fully understood. In the following sections, we describe the key pre- and postnatal factors that have been shown to affect the pathogenesis of NAFLD in children.

\section{Prenatal Factors}

Recent evidence has shown that there are some prenatal factors that are responsible for the pathogenesis of pediatric NAFLD, such as maternal obesity, metabolic syndrome during pregnancy, gestational diabetes, and low birth weight. ${ }^{43-46}$ This is supported by a series of well-conducted in vitro and in vivo mechanistic studies. It is a multifactorial process that results from a combination of biochemical factors (fetal insulin, lipid profile) and epigenetic modification, which influences hepatic DNL, mitochondrial function, and oxidative stress in hepatocytes, macrophages, and adipocytes. ${ }^{47-53}$ It is generally agreed that a "second hit" is required to initiate development of NAFLD as an adolescent or adult, for which the most important is a positive energy balance in the form of a high-fat/high-carbohydrate (Western) diet. ${ }^{46,51,52,54}$ Recent human studies showed that there is an association between maternal prepregnancy BMI and infant ectopic fat deposition in the liver and in the intra-abdominal cavity ${ }^{45}$ (- Table 1). Magnetic resonance imaging (MRI) has been used to measure adiposity and hepatic liver fat in newborns of obese mothers and women with gestational diabetes. ${ }^{44,45}$ In one of these studies, a correlation was shown between gestational BMI and offspring hepatic lipid accumulation. ${ }^{45}$ Though it is unclear whether these same newborns go on to develop progressive NAFLD, a possible explanation for the ectopic hepatic fat deposition in these newborns may be that immature fetal adipocytes are not sufficiently developed to accommodate and store lipids crossing the placenta in excess, throughout pregnancy. Consequently, in the presence of maternal obesity or gestational diabetes mellitus (two states where nonesterified fatty acid concentrations might be expected to be increased), excess transference of maternal lipid will result in accumulation of fetal ectopic fat as the fetus is not able to expand adipose depots to buffer the increased transplacental lipid delivery. ${ }^{55}$ The presence and persistence of liver fat after birth have also been shown by us in a mouse model developed to investigate developmental programming of offspring NAFLD. ${ }^{56}$ In this study, we showed that increased dietary maternal fat intake in the mother from before conception primed the development of increased liver fat in the offspring mice in adulthood, even if the offspring mice had only ever eaten a normal chow (carbohydrate-rich, low-fat) diet from weaning until adulthood. Furthermore, adult mice that had been exposed in utero to increased dietary maternal fat intake in the mother from before conception, coupled with only consuming the same high-fat diet from weaning until adulthood, developed a florid form of NASH, which was a considerably more severe form of NAFLD than comparator mice who had only been exposed to the high-fat diet from weaning until adulthood. Additionally, mice exposed to gestational high-fat diet had decreased mitochondrial electron transport chain function, suggesting that a stressor in early live caused dysfunctional mitochondria that are less efficient at preforming mitochondrial $\beta$-oxidation, predisposing the mouse to hepatic fat accumulation and programming the development of NAFLD in adulthood. Another interesting prenatal factor associated with the pathogenesis of pediatric NAFLD is the perturbation of intrauterine environment during pregnancy. According to the "thrifty phenotype" hypothesis, intrauterine growth retardation can lead to several chronic conditions and metabolic disorders (such as NAFLD) later in life. ${ }^{57}$ We have shown that the "small for gestational age" state was significantly associated with severe liver steatosis (NAFLD Activity Score $>5$ ) in childhood. ${ }^{58}$ Moreover, in a large epidemiological study, Sandboge et a have also shown that body weight at 2 years was negatively associated with NAFLD after adjusting for age, sex, and gestational age. Thus, the current evidence to date suggests that the intrauterine environment has the potential to have a powerful effect on the offspring's future risk of developing NAFLD later in life.

\section{Postnatal Factors}

The postnatal factors predisposing to pediatric NAFLD are similar to those for adult NAFLD and include common factors such as obesity, hyperinsulinemia, and insulin resistance. In the presence of obesity, the potential for good adipose tissue expansion is fundamental for maintaining lipid homeostasis and insulin sensitivity and for protecting the lipid from increased lipid and inflammatory fluxes that have the potential for promoting development and subsequent progression of NAFLD. When the process of the adipose tissue expansion fails, there is an associated low-grade inflammation 
Pediatric NAFLD Mann et al.

Table 1 Human evidence for prenatal and infant risk factors associated with NAFLD

\begin{tabular}{|c|c|c|}
\hline Study & Methodology & Findings \\
\hline Ayonrinde et al $^{123}$ & $\begin{array}{l}N=1,170 \\
\text { Mean age: } 17 \text { y } \\
\text { Prospective } \\
\text { USS-diagnosed NAFLD }\end{array}$ & $\begin{array}{l}\text { NAFLD independently associated with: } \\
\text { Maternal prepregnancy obesity } \\
\text { (OR: } 2.3 \text { ) } \\
\text { Breastfeeding } 64 \text { mo (OR: } 0.6) \\
\text { Adolescent obesity (OR: } 9.1)\end{array}$ \\
\hline $\begin{array}{l}\text { Bugianesi et al:; }{ }^{58} \text { Nobili et al; }{ }^{124} \text { and } \\
\text { Nobili et } \mathrm{al}^{25}\end{array}$ & $\begin{array}{l}N=288 \\
\text { Mean age: } 13 \text { y } \\
\text { Retrospective } \\
\text { Biopsy-diagnosed NAFLD }\end{array}$ & $\begin{array}{l}\text { Low birth weight associated with } \\
\text { increased severe steatosis and portal } \\
\text { inflammation, independent of insulin } \\
\text { resistance } \\
\text { Each month of breastfeeding reduced } \\
\text { NASH (OR: } 0.7 \text { ) and fibrosis (OR: } 0.9 \text { ) } \\
\text { SGA associated with insulin resistance }\end{array}$ \\
\hline Suomela et $a^{23}$ & $\begin{array}{l}N=2,042 \\
\text { Mean age: } 42 \text { y } \\
\text { Prospective } \\
\text { USS-diagnosed NAFLD }\end{array}$ & $\begin{array}{l}\text { NAFLD independently associated with: } \\
\text { Preterm birth (OR: } 2.4) \\
\text { Small for gestation age (OR: } 1.8) \\
\text { Birth weight (OR: } 0.8) \\
\text { Insulin at } 11 \text { y (OR: } 1.3 \text { ) }\end{array}$ \\
\hline Breij et al ${ }^{125}$ & $\begin{array}{l}N=268 \\
\text { Mean age: } 21 \text { y } \\
\text { Retrospective } \\
\text { FLI-diagnosed NAFLD }\end{array}$ & $\begin{array}{l}\text { Rapid catch-up growth in the first } 3 \text { mo } \\
\text { associated with high FLI score } \\
\text { No association of FLI with SGA }\end{array}$ \\
\hline Sandboge et al ${ }^{126}$ & $\begin{array}{l}N=1,587 \\
\text { Mean age: } 62 \text { y Retrospective } \\
\text { NAFLD liver fat score/equation diag- } \\
\text { nosed NAFLD }\end{array}$ & $\begin{array}{l}\text { Weight at } 2 \text { y negatively correlated with } \\
\text { NAFLD score } \\
\text { Low weight at } 2 \text { y with later adult obe- } \\
\text { sity had high risk of NAFLD (OR: 19.5) }\end{array}$ \\
\hline Fraser et $\mathrm{al}^{127}$ & $\begin{array}{l}N=2,101 \\
\text { Mean age: } 68 \text { y } \\
\text { Retrospective } \\
\text { Abnormal LFT }\end{array}$ & $\begin{array}{l}\text { Birth weight negatively correlated with } \\
\text { ALT, GGT, and ALP }\end{array}$ \\
\hline
\end{tabular}

Abbreviations: ALT, alanine aminotransferase; AST; aspartate aminotransferase; FLI, fatty liver index; GGT, gamma-glutamyl transpeptidase; LFT, liver function test; NAFLD, nonalcoholic fatty liver disease; NASH, nonalcoholic steatohepatitis; OR, odds ratio; SGA, small for gestational age; USS, ultrasound scan.

associated with adipocyte autophagy and adipokine production. In this low-grade inflammatory state, there is increased flux of free fatty acids from the adipose tissue to the liver together with increased proinflammatory cytokines causing the recruitment of macrophages in adipose tissue, stimulating the production of TNF- $\alpha$, IL- 6 , and reactive oxygen species, and increasing adipocyte lipolysis. ${ }^{59}$ In the liver, the combined effect of free fatty acids and adipokines coming from the adipose tissue increases endoplasmic reticulum stress with consequent activation of Kupffer cells, and this triggers liver inflammation, potentially promoting development of NASH. ${ }^{60}$ This inflammatory state triggers a cascade of hepatocyte injury and increases oxidative stress and mitochondrial dysfunction with consequent impairment of liver metabolic capacity. Hyperinsulinemia and insulin resistance also contribute to the development of NAFLD in adolescence as during puberty, children experience a physiological state of insulin resistance. In a large cross-sectional study, Moran et al studied 357 healthy children and adolescents who underwent hyperinsulinemic-euglycemic clamps. They showed that there are significant differences in insulin resistance between boys and girls and that insulin resistance increases significantly at Tanner stages 2,3 , and 4 but decreases to near prepubertal levels at Tanner stage 5 .
Furthermore, while insulin resistance was related to BMI and anthropometric measures of fatness, these factors did not completely explain the insulin resistance that occurs during the Tanner stages of puberty. ${ }^{61}$ Moreover, in further work, the authors showed there are sex-related developmental changes in insulin resistance, which are independent of changes in adiposity. ${ }^{62}$ During the transition from late childhood through adolescence, insulin resistance in males increased in association with increased triglyceride concentrations and decreased high-density lipoprotein cholesterol levels. This phenomenon was noted despite a concurrent reduction in body fatness in male children, whereas the opposite effect was observed in female children. It is interesting to speculate that these sex-related developmental changes in insulin resistance may underpin not only differences in NAFLD prevalence between males and females in adulthood but also differences in cardiovascular risk between males and females in adult life.

In childhood, a physiological state of insulin resistance plus a sedentary lifestyle and the consumption of unhealthy food $^{63}$ may increase obesity, decrease skeletal muscle oxidation of lipids, and promote hepatic DNL. In the presence of hyperinsulinemia that is associated with insulin resistance, acetyl-CoA carboxylase is activated by insulin, and acetyl- 
CoA is converted into malonyl-CoA, which is the committed step in hepatic fatty acid synthesis. There are two important transcription factors that regulate DNL: sterol regulatory element-binding protein-1 (SREBP-1c) and carbohydrate response element-binding protein (ChREBP). These transcription factors regulate enzymes involved in fatty acid and triacyl glycerol synthesis. In addition, a high-carbohydrate diet, particularly one that contains high levels of dietary fructose, can increase hepatic DNL by increasing substrate supply and promoting the expression of SREBP1c and ChREBP. ${ }^{64,65}$ DNL is an energy-expensive process consuming 7 ATPs and 14 NADPH to generate each palmitate from acetyl-CoA. ${ }^{66}$ Consistent with this, fructose causes hepatic ATP depletion with resultant oxidative stress and potential for mitochondrial dysfunction. ${ }^{67,68}$

Softic et al investigated the differential effects of glucose and fructose in a mouse model and showed that the addition of fructose to a high-fat diet was associated with increased expression of SREBP-1c and ChREBP and increased fatty acid synthesis, as well as hepatic insulin resistance. In contrast, when glucose was added to the high-fat diet, the authors found an increase in total ChREBP and liver triglyceride accumulation but not insulin resistance (- Fig. 1). ${ }^{64,69}$ In the same study, Softic et al studied hepatic expression of ketohexokinase (KHK), an enzyme that catalyzes the first step of intracellular fructose metabolism, ${ }^{70}$ in both mice and obese adolescents with NAFLD who were undergoing bariatric surgery. The authors found that KHK expression was increased twofold in mice whose high-fat diet was supplemented with fructose. This finding contrasted with their observations in mice whose high-fat diet was supplemented with glucose, where KHK expression did not increase significantly. In the adolescents, KHK expression was twofold higher in obese patients with NASH compared with obese patients without fatty liver. Thus, these data suggest that dietary fructose is associated with an increased expression of KHK favoring the production of acyl-CoA that contributes to the development of NAFLD through increased DNL. ${ }^{69}$ The high activity of KHK contributes to reduced cellular ATP by rapid phosphorylation of fructose, and, through regeneration of ATP in oxidative phosphorylation, hepatocytes become depleted in inorganic phosphate. The net effect is elevated uric acid ${ }^{71}$ production, which has been independently associated with advanced NASH histology in children. ${ }^{13}$

\section{Hepatic Outcomes}

The long-term hepatic outcomes of pediatric NAFLD are not clear due to a lack of prospective natural history data, and few children undergo paired biopsies. The outcomes of NAFLD in adults are more defined, where data demonstrate that after over 30 years of follow-up, NAFLD is associated with increased all-cause mortality (hazard ratio [HR]: 1.3) and HCC in (OR: 6.6). ${ }^{30}$ Overall, liver-related events occur in $<10 \%$ of patients with NAFLD, but there is a strong correlation with fibrosis stage and outcome. ${ }^{29}$ In addition, it has been suggested that

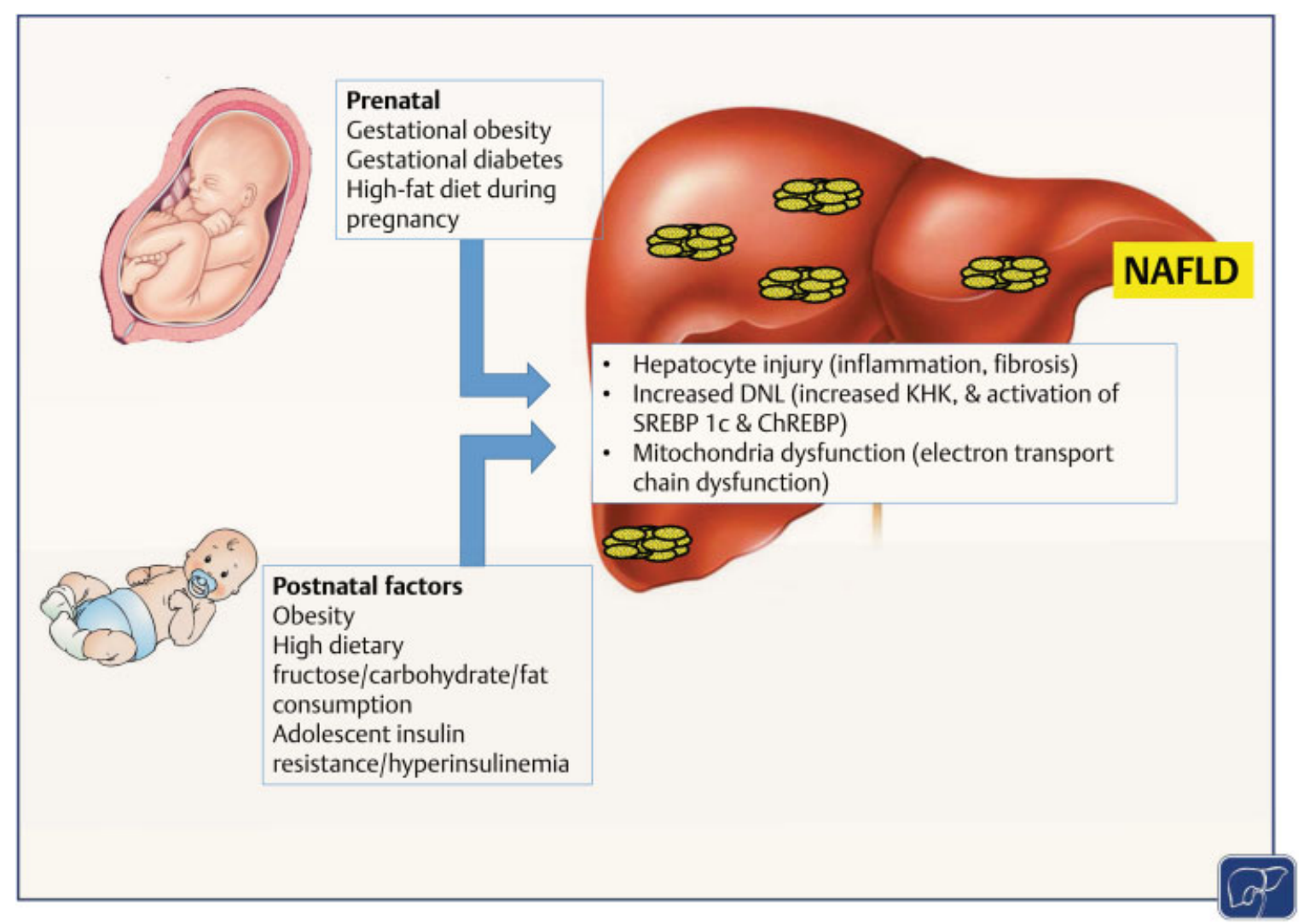

Fig. 1 Pre- and postnatal factors contribute in NAFLD pathogenesis. 
there is a group of "rapid progressors" who may develop severe fibrosis within 5 years, ${ }^{28}$ though it remains to be established whether this is due to sampling variability on repeat biopsy. Liver-related causes of death are third after cardiovascular disease and nonhepatic malignancy.

There are no robust data to determine whether this natural history holds true for pediatric NAFLD. It is also not well established what proportion of children with NAFLD continue to have NAFLD as adults. It is known that around a third of obese adolescents will become obese adults, ${ }^{72}$ but a follow-up study from pediatric to adult transition in NAFLD has not yet been reported.

Feldstein et al reported a retrospective cohort of 66 children with NAFLD in which 2 children underwent transplant for decompensated cirrhosis. ${ }^{73}$ This study has the longest followup, but its retrospective design and lack of data on method of diagnosis for children limit its conclusions. There have been other case series to suggest that severe fibrosis is possible while still a child, ${ }^{74}$ however, it is generally regarded that endstage liver disease while under 16 years of age is unlikely to be secondary to NAFLD alone and should prompt a search for alternative diagnoses. ${ }^{75}$ It is worthy to mention that there are some specific circumstances, particularly acquired hypothalamic-pituitary insufficiency, that may be associated with rapid progression of fibrosis. ${ }^{76}$

In adults, fibrosis is the sole predictor of long-term liverrelated events. ${ }^{31}$ This finding has been reproduced in multiple cohorts, and noninvasive fibrosis scores (NAFLD fibrosis score, Fibrosis 4, BARD [BMI $\geq 28 \mathrm{~kg} / \mathrm{m}^{2}$, an AST/ALT ratio $\geq 0.8$ and diabetes]) correlate with mortality. ${ }^{77}$ While similar scores exist in pediatric NAFLD (e.g., the pediatric NAFLD fibrosis index ${ }^{78}$ ), it is not known whether they correlate with long-term outcome.

Data from randomized controlled trials demonstrate that stage 1 and 2 fibroses are readily reversible within 2 years. ${ }^{79,80}$ It is not known to what extent advanced fibrosis can regress in pediatric NAFLD.

Therefore, to accurately determine the long-term hepatic outcomes in pediatric NAFLD, natural history studies are required. These have recently been established on both sides of the Atlantic ${ }^{81,82}$ and are likely to require more than 20 years follow-up to quantify rates of HCC development and liver failure.

\section{Dangerous Liaisons}

There are several potential pitfalls in the diagnosis, monitoring, and management of pediatric NAFLD, which will be discussed in this section.

The most important point about diagnosing NAFLD in children is to exclude conditions masquerading as fatty liver. It is recognized that a variety of liver and systemic disorders may cause secondary steatosis, including those listed in - Table 2.

Schwimmer et al presented strong evidence for liver biopsy in children with suspected NAFLD. Out of 374 children referred from primary care, 255 underwent biopsy and 61 had a diagnosis other than NAFLD, most frequently autoimmune hepatitis. Children and adults with "lean" NAFLD, with BMI and waist circumference $<95$ percentile, are more likely to have secondary causes for steatosis or NAFLD-associated polymorphisms. ${ }^{83}$ In very lean children with severe insulin resistance, lipodystrophy should be considered. ${ }^{84}$

Differentiating between WD and NAFLD may be very challenging, and some pediatricians would argue that biopsy is the only method to truly exclude WD. Ceruloplasmin is a good screening test for WD and, depending on the threshold used, may have a negative predictive value of $99 \%$ ( - Table 3) ${ }^{85}$ However, a subset of patients may have normal ceruloplasmin, and missing the diagnosis can result in permanent neurologic damage. Therefore, a liver biopsy with dry weight copper improves the reliability of diagnosis, but can give a false-positive result (for WD) as cholestatic disorders can cause copper accumulation. ${ }^{75}$ Molecular genetics can be used to make a diagnosis of WD, but ATP7B can be affected by a variety of mutations; therefore, genetics cannot exclude the diagnosis. ${ }^{86}$

Lysosomal acid lipase deficiency (LAL-D), also known as cholesterol ester storage disease, is a potential differential

Table 2 Some secondary causes of hepatic steatosis that should be excluded before making a diagnosis of pediatric NAFLD

\begin{tabular}{|l|l|l|}
\hline Secondary causes of NAFLD & Test & Abnormality \\
\hline Hepatitis B and C, EBV, CMV & Viral hepatitis serology & IgG or IgM serology positive \\
\hline \multirow{2}{*}{ Wilson's disease } & Serum ceruloplasmin & Low levels \\
\cline { 2 - 3 } & Liver biopsy & Raised dry weight copper \\
\hline \multirow{2}{*}{ Autoimmune hepatitis } & Autoantibodies & Positive ANA/ASMA \\
\cline { 2 - 3 } & Immunoglobulins & Increased IgG \\
\hline Alpha-1-antitrypsin deficiency & Alpha-1-antitrypsin levels and Pi type & PiZZ \\
\hline Hereditary hemochromatosis & Serum iron studies & Raised ferritin and transferrin saturation \\
\hline \multirow{2}{*}{$\begin{array}{l}\text { Lipodystrophy and other insulin-resis- } \\
\text { tance }\end{array}$} & Clinical examination & Reduced subcutaneous adipose \\
\cline { 2 - 3 } & Serum fasting insulin & Greatly elevated \\
\hline LAL deficiency & Dry blood spot assay & Reduced LAL activity \\
\hline
\end{tabular}

Abbreviations: ANA, antinuclear antibodies; ASMA, antismooth muscle antibodies; CMV, cytomegalovirus; EBV, Epstein-Barr virus; IgG, immunoglobulin G; IgM, immunoglobulin M; LAL, lysosomal acid lipase; NAFLD, nonalcoholic fatty liver disease. 
Pediatric NAFLD Mann et al. 7

Table 3 Data on the validity of use of three different cutoff thresholds of ceruloplasmin for the diagnosis of Wilson's disease in patients clinically suspected of having the condition

\begin{tabular}{|l|l|l|l|l|}
\hline $\begin{array}{l}\text { Ceruloplasmin } \\
\text { concentration } \\
\text { cutoff }(\mathbf{g} / \mathbf{L})\end{array}$ & Sensitivity (\%) & Specificity (\%) & $\begin{array}{l}\text { Positive predictive } \\
\text { value (\%) }\end{array}$ & $\begin{array}{l}\text { Negative predictive } \\
\text { value (\%) }\end{array}$ \\
\hline 0.20 & 98 & 56 & 48 & 99 \\
\hline 0.14 & 93 & 100 & 100 & 97 \\
\hline 0.10 & 79 & 100 & 100 & 92 \\
\hline
\end{tabular}

diagnosis for NAFLD. This rare, autosomal recessive lysosomal disorder has a wide spectrum of clinical presentation. ${ }^{87}$ Wolman disease is the most severe form, with rapidly progressive liver failure in infants. A milder form presents in childhood or adults with hepatic steatosis, raised aminotransferases, raised low-density lipoprotein, and low highdensity lipoprotein. Diagnosis is theoretically important because of the potential for treatment with sebelipase alfa. ${ }^{88}$ However, despite the possibility of misdiagnosis of LAL-D as NAFLD, or a role of LAL in NAFLD, ${ }^{89}$ to date there are no reports of identification of LAL-D in cohorts of children with fatty liver; therefore, its relevance to routine clinical practice remains to be established.

It is not clear how best to monitor pediatric NAFLD, but it is well established that aminotransferases are a poor marker of disease activity. Alanine aminotransferase (ALT) and aspartate aminotransferase fluctuate throughout the disease course and do not correlate with fibrosis, ${ }^{90}$ though they may show some correlation with NAFLD activity score. ${ }^{38}$ Blood often becomes most abnormal during the relatively insulinresistant state of adolescence; ${ }^{91}$ therefore, a reduction of ALT without evidence of weight loss should not be relied upon as a reassuring finding.

Though the long-term cardiovascular and metabolic outcomes of pediatric NAFLD have yet to be formally quantified, these are likely to be the main burden of disease for patients in adult life. ${ }^{29,32}$ Therefore, hepatologists must remember that weight loss and improvement of insulin resistance are the most important management goals. Apart from therapies, targets at improving liver disease activity are discussed below.

\section{Management Program}

The treatment of pediatric NAFLD can be divided into conservative, medical, and surgical approaches. As alluded to above, the determinants of successful management are not clear as the natural history of the condition is uncertain. The goals are multidimensional: improve the metabolic health of children to reduce their long-term cardiovascular risk and reduce liver-related clinical events, presumably by targeting fibrosis. Results from the major controlled trials in pediatric NAFLD are summarized in - Table 4.

Weight loss is the core therapy for pediatric NAFLD and for all children with obesity. ${ }^{92}$ This may be achieved by dietary modification and/or physical activity. A variety of diets have been used in randomized and nonrandomized clinical trials.
The most frequently used diet is a "low-fat" hypocaloric diet with 25 to $30 \mathrm{kcal} / \mathrm{kg}, 50$ to $60 \%$ carbohydrate and 23 to $30 \%$ fat (with one-third saturated fat). ${ }^{80,93,94} \mathrm{~A}$ recent systematic review of dietary and physical activity interventions found that there was insufficient evidence to suggest any single method of weight loss, but that greater weight loss gave a larger improvement in noninvasive markers of NAFLD. ${ }^{95}$ In studies with biopsy endpoints, a reduction in age- and sexcorrected BMI is associated with an improvement in features of NAS and fibrosis. ${ }^{80}$

There is recent physiological evidence to suggest that fructose restriction improves the metabolic profile of obese children and potential for treatment of fatty liver. ${ }^{12}$ Lowcarbohydrate $^{96}$ or low-fructose ${ }^{97}$ diet has only been used in pilot studies on pediatric NAFLD; however, there are several larger studies planned. ${ }^{98}$

However, it must be remembered that even with close follow-up in well-conducted clinical trials, there is a relatively poor response to dietary and physical activity in childhood obesity. ${ }^{99}$ Compliance is poor, and participants with low uptake of advice have greater increases in aminotransferases. ${ }^{93}$ Therefore, NAFLD should ideally be managed in a multidisciplinary clinic that includes a dietician, clinical nurse specialist, and clinical psychologist to give the best chance of achieving weight loss.

There are no approved pharmacological therapies of pediatric NAFLD. ${ }^{100}$ The main groups of agents that have been tested to date are antioxidants, metformin, polyunsaturated fatty acids (PUFAs), probiotics, and vitamin D.

The American Association for the Study of Liver Diseases (AASLD) guidance recommends vitamin $\mathrm{E}$ as the only therapy that is potentially efficacious in pediatric NAFLD. ${ }^{92}$ This is primarily based on data from the Treatment of NAFLD in Children (TONIC) trial that demonstrated an improvement in ballooning and overall NAS with vitamin E use, without any major adverse events. ${ }^{79}$ Other studies have used vitamin $\mathrm{E}$ in a variety of doses and durations but have been limited by not using protocolled paired biopsies, ${ }^{93,101}$ combination treatment, ${ }^{80,102}$ or differing weight loss between intervention arms. ${ }^{103}$ Overall, vitamin $\mathrm{E}$ is probably safe and may improve NASH activity, though it remains to be established if this translates into improved liver-related outcomes.

Results from a randomized trial of cysteamine bitartrate delayed release (CBDR) have recently shown modest improvements in lobular inflammation, and there was overall improvement in histology in participants weighing under $65 \mathrm{~kg} .{ }^{104}$ It acts by increasing intracellular glutathione, 
8 Pediatric NAFLD Mann et al.

Table 4 Randomized controlled trials in pediatric NAFLD

\begin{tabular}{|c|c|c|c|}
\hline Trial & $N$ & Intervention & End points \\
\hline \multicolumn{4}{|l|}{ Dietary intervention } \\
\hline Vos et al ${ }^{97}$ & 10 & Low-fructose vs. low-fat diet & Liver enzymes: no effect \\
\hline $\begin{array}{l}\text { Ramon-Krauel } \\
\text { et al }\end{array}$ & 16 & Low-glycemic-load vs. low-fat diet & $\begin{array}{l}\text { Liver enzymes and MRS: improved but no dif- } \\
\text { ference between groups }\end{array}$ \\
\hline Jin et $a^{128}$ & 21 & Low-fructose vs. standard diet & Liver enzymes and MRS: no effect \\
\hline \multicolumn{4}{|l|}{ Antioxidants } \\
\hline Vajro et $\mathrm{al}^{93}$ & 28 & Lifestyle vs. lifestyle + vitamin $\mathrm{E}$ & $\begin{array}{l}\text { Liver enzymes: improved but no difference } \\
\text { between groups } \\
\text { US: unchanged }\end{array}$ \\
\hline Nobili et $\mathrm{al}^{80}$ & 53 & Lifestyle vs. lifestyle + vitamin $E+$ vitamin $C$ & $\begin{array}{l}\text { Histological steatosis, lobular inflammation, } \\
\text { ballooning, and NAS: improved but no differ- } \\
\text { ence between groups }\end{array}$ \\
\hline Wang et al ${ }^{103}$ & 76 & $\begin{array}{l}\text { No intervention vs. strict lifestyle vs. unstruc- } \\
\text { tured lifestyle + vitamin E. }\end{array}$ & $\begin{array}{l}\text { Liver enzymes: improved with strict lifestyle } \\
\text { intervention } \\
\text { US: unchanged }\end{array}$ \\
\hline Lavine et $\mathrm{al}^{79}$ & 173 & Metformin vs. vitamin E vs. placebo & $\begin{array}{l}\text { NAS and ballooning improved with vitamin E } \\
\text { Liver enzymes: improved but no difference } \\
\text { between groups }\end{array}$ \\
\hline Akcam et al ${ }^{101}$ & 67 & $\begin{array}{l}\text { Lifestyle vs. lifestyle + metformin vs. lifestyle } \\
+ \text { vitamin E }\end{array}$ & $\begin{array}{l}\text { Liver enzymes: improved but no difference } \\
\text { between groups }\end{array}$ \\
\hline Shiasi Arani et al ${ }^{105}$ & 119 & Metformin vs. vitamin E vs. placebo & US: improved but no difference between drugs \\
\hline Schwimmer et al ${ }^{104}$ & 169 & CBDR vs. placebo & $\begin{array}{l}\text { Lobular inflammation: improved } \\
\text { Liver enzymes: improved }\end{array}$ \\
\hline Zöhrer et al ${ }^{102}$ & 40 & Lifestyle vs. lifestyle + DHA-Cho-VE & $\begin{array}{l}\text { Liver enzymes and US: improved with DHA-Cho-VE } \\
\text { Histological improvement in DHA-Cho-VE, but no } \\
\text { biopsy in placebo for comparison }\end{array}$ \\
\hline \multicolumn{4}{|l|}{ Metformin } \\
\hline Nadeau et al ${ }^{106}$ & 50 & Lifestyle vs. lifestyle + metformin & $\begin{array}{l}\text { Liver enzymes: improved but no difference } \\
\text { between groups } \\
\text { US: improved with metformin }\end{array}$ \\
\hline \multicolumn{4}{|c|}{ Polyunsaturated fatty acids } \\
\hline Nobili et al ${ }^{108}$ & 60 & DHA vs. placebo & Liver enzymes and US steatosis: improved \\
\hline Boyraz et $\mathrm{al}^{94}$ & 108 & Lifestyle vs. lifestyle + PUFA & $\begin{array}{l}\text { Liver enzymes and US: improved in lifestyle + } \\
\text { PUFA group }\end{array}$ \\
\hline Janczyk et al ${ }^{129}$ & 76 & DHA/EPA vs. placebo & $\begin{array}{l}\text { Liver enzymes and US: improved but no differ- } \\
\text { ence between groups }\end{array}$ \\
\hline Pacifico et al ${ }^{109}$ & 51 & DHA vs. placebo & $\begin{array}{l}\text { Liver enzymes: improved but no difference } \\
\text { between groups } \\
\text { MRI hepatic fat: improved }\end{array}$ \\
\hline Della Corte, et al ${ }^{117}$ & 41 & $\mathrm{DHA}+$ vitamin D vs. placebo & $\begin{array}{l}\text { Liver enzymes: improved } \\
\text { Histological improvement in DHA + Vitamin D, } \\
\text { but no biopsy in placebo for comparison }\end{array}$ \\
\hline \multicolumn{4}{|l|}{ Probiotics } \\
\hline Vajro et al ${ }^{130}$ & 20 & Lactobacillus vs. placebo & $\begin{array}{l}\text { Liver enzymes: improved } \\
\text { US: unchanged }\end{array}$ \\
\hline Alisi et al ${ }^{110}$ & 44 & Lifestyle vs. lifestyle + VSL\#3 & $\begin{array}{l}\text { Liver enzymes: unchanged } \\
\text { US: improved with VSL\#3 }\end{array}$ \\
\hline Famouri et al ${ }^{111}$ & 64 & Prokid probiotic vs. placebo & Liver enzymes and US steatosis: improved \\
\hline \multicolumn{4}{|l|}{ Bariatric surgery } \\
\hline Manco et al ${ }^{118}$ & 93 & $\begin{array}{l}\text { Sleeve gastrectomy vs. IGWLD vs. lifestyle } \\
\text { (nonrandomized) }\end{array}$ & $\begin{array}{l}\text { Fibrosis and NASH: improved most in sleeve } \\
\text { gastrectomy as well as in IGWLD }\end{array}$ \\
\hline
\end{tabular}

Abbreviations: CBDR, cysteamine bitartrate delayed release; DHA, docosahexaenoic acid; DHA-Cho-VE, docosahexaenoic acid with choline and vitamin E; EPA, eicosapentaenoic acid; IGWLD, intragastric weight loss device; MRS, magnetic resonance spectroscopy; NAFLD, nonalcoholic fatty liver disease; NAS, NAFLD activity score; NASH, nonalcoholic steatohepatitis; PUFA, polyunsaturated fatty acids; US, ultrasound; VSL\#3, a probiotic mixture. 
which then scavenges oxygen free radicals. The evidence body for CBDR is smaller than that for vitamin E, and it is likely that further studies will be required before it is incorporated into guidelines.

Metformin has had more modest results, with some reports of improved radiological evidence of steatosis, ${ }^{105,106}$ but randomized biopsy data are limited than those from the TONIC trial. ${ }^{79}$ However, aside from its effects on the liver, metformin may be used by pediatric endocrinologists in children with insulin resistance and thus at a risk of type 2 diabetes. ${ }^{99,107}$ At this time, consensus guidelines do not recommend metformin as a primary treatment for NAFLD or NASH.

PUFAs, mostly docosahexaenoic acid (DHA) and eicosapentaenoic acid, are given with the aim of alerting the composition of the hepatic lipidome and reducing lipotoxicity. Noninvasive data are encouraging, where use of at least $250 \mathrm{mg}$ per day for 6 months may result in reduction of radiological steatosis, but there are no biopsy data available to confirm these findings. ${ }^{94,108,109}$

The evidence of use of probiotics is similar: a variety of regimens have been used in trials for pediatric NAFLD, but there are no studies with biopsy endpoints to date. ${ }^{10,111}$ Although there is a large body of evidence that confirms the association of intestinal dysbiosis with obesity and NAFLD, ${ }^{12-114}$ there are little data to support modulation of the microbiome as a primary treatment strategy.

The association of vitamin $\mathrm{D}$ deficiency with pediatric obesity, ${ }^{115}$ NAFLD, and NASH ${ }^{116}$ is relatively well established, and the use of replacement therapy in deficient children is logical. Indeed, in children deficient in vitamin $\mathrm{D}$, replacement in combination with DHA does improve histology. ${ }^{117}$ However, it remains to be demonstrated whether children with acceptable $25-\mathrm{OH}-\mathrm{D}_{3}(>20 \mathrm{ng} / \mathrm{L})$ benefit from supplementary vitamin $D$. In addition, the risk of hypervitaminosis and resulting renal impairment must be considered.

Finally, bariatric surgery is a treatment option for severe pediatric obesity with comorbidities. Expert consensus recommends that NAFLD should not be a primary indication for weight loss surgery; however, evidence does suggest it to be of use, with potential for reversal of fibrosis. ${ }^{118,119}$ These findings are consistent with data from adults, though there were insufficient unbiased studies for a Cochrane review to recommend it as a primary treatment. ${ }^{120}$ However, the longterm data are clear that bariatric surgery improves the metabolic and cardiovascular outcomes for obese individuals, ${ }^{121}$ and therefore it may be part of the care for patients with NAFLD. The psychological effects of bariatric surgery are not to be underestimated, and there are emerging data on the impact that such operations have on patients later in adult life. ${ }^{122}$

Therefore, weight loss is the primary treatment of pediatric NAFLD, and it appears that the method by which this is achieved does not affect the outcome. Vitamin E is the only drug treatment that may be a direct hepatic benefit, but pharmacological management of the liver is a small component of the overall care for children with the metabolic syndrome.

\section{Implications and Future Directions}

The implications of these data are that pediatric NAFLD is increasing, and that these patients will become adults with end-stage liver disease or HCC in 10 to 30 years' time. Weight loss is the only intervention demonstrated to have significant efficacy. Further investigation into the pathogenesis of pediatric NAFLD is needed to complement translational studies, with an aim to develop novel therapeutic strategies. There are only a few agents in trials in children, and drug development is progressing at a much slower rate than in adults.

Future directions must focus on establishing and expanding our understanding of NAFLD pathogenesis with a translational therapy in mind, for example, determining the microbiome phylogeny associated with less severe fibrosis and trialing probiotic therapy. It may also involve exploring whether recent advances in adult hepatology are also applicable to pediatrics, such as the role of VAP-1 in gut-liver axis, which has not yet been investigated in children. There is much heterogeneity in investigations of NAFLD, particularly in regard to the use of biopsy, and establishing biomarkers that correlate with histology progression and clinical outcomes remains a major target. Finally, despite weight loss being the primary treatment for NAFLD, minimal progress has been made in guidance on determining the optimal regimen of lifestyle changes.

In conclusion, despite major advances in understanding, research in pediatric NAFLD has not yet translated into patient benefit. It is an important condition with unmet scientific and clinical needs that requires urgent attention to slow the future epidemic of end-stage liver disease in adults.

\section{Abbreviations}

NAFLD nonalcoholic fatty liver disease

NAFL nonalcoholic fatty liver

NASH nonalcoholic steatohepatitis.

Conflict of Interest

All authors declare that there is no conflict of interest that could be perceived as affecting the impartiality of the reported research.

\section{Funding}

No external funding supported the research described in this manuscript. This research did not receive any specific grant from any funding agency in the public, commercial, or not-for-profit sector.

\section{Authors' Contribution}

All authors equally participated to the manuscript, approved the final version as submitted, and agreed to be accountable for all aspects of the work.

\section{References}

1 Nobili V, Svegliati-Baroni G, Alisi A, Miele L, Valenti L, Vajro P. A 360-degree overview of paediatric NAFLD: recent insights. J Hepatol 2013;58(06):1218-1229 
2 Mencin AA, Lavine JE. Nonalcoholic fatty liver disease in children. Curr Opin Clin Nutr Metab Care 2011;14(02):151-157

3 Schwimmer JB, Deutsch R, Kahen T, Lavine JE, Stanley C, Behling C. Prevalence of fatty liver in children and adolescents. Pediatrics 2006;118(04):1388-1393

4 Pacifico L, Poggiogalle E, Cantisani V, et al. Pediatric nonalcoholic fatty liver disease: a clinical and laboratory challenge. World J Hepatol 2010;2(07):275-288

5 Anderson EL, Howe LD, Jones HE, Higgins JP, Lawlor DA, Fraser A. The prevalence of non-alcoholic fatty liver disease in children and adolescents: a systematic review and meta-analysis. PLoS One 2015;10(10):e0140908

6 Gaggini M, Morelli M, Buzzigoli E, DeFronzo RA, Bugianesi E, Gastaldelli A. Non-alcoholic fatty liver disease (NAFLD) and its connection with insulin resistance, dyslipidemia, atherosclerosis and coronary heart disease. Nutrients 2013;5(05): $1544-1560$

7 Valenti L, Bugianesi E, Pajvani U, Targher G. Nonalcoholic fatty liver disease: cause or consequence of type 2 diabetes? Liver Int 2016;36(11):1563-1579

8 Nobili V, Liccardo D, Bedogni G, et al. Influence of dietary pattern, physical activity, and I148M PNPLA3 on steatosis severity in atrisk adolescents. Genes Nutr 2014;9(03):392

9 Dongiovanni P, Valenti L. Genetics of nonalcoholic fatty liver disease. Metabolism 2016;65(08):1026-1037

10 Dongiovanni P, Lanti C, Riso P, Valenti L. Nutritional therapy for nonalcoholic fatty liver disease. J Nutr Biochem 2016;29:1-11

11 Vos MB, Lavine JE. Dietary fructose in nonalcoholic fatty liver disease. Hepatology 2013;57(06):2525-2531

12 Schwarz JM, Noworolski SM, Erkin-Cakmak A, et al. Effects of dietary fructose restriction on liver fat, de novo lipogenesis, and insulin kinetics in children with obesity. Gastroenterology 2017; 153(03):743-752

13 Mosca A, Nobili V, De Vito R, et al. Serum uric acid concentrations and fructose consumption are independently associated with NASH in children and adolescents. J Hepatol 2017;66(05): 1031-1036

14 Santoro N, Savoye M, Kim G, et al. Hepatic fat accumulation is modulated by the interaction between the rs738409 variant in the PNPLA3 gene and the dietary omega6/omega3 PUFA intake. PLoS One 2012;7(05):e37827

15 Romeo S, Kozlitina J, Xing C, et al. Genetic variation in PNPLA3 confers susceptibility to nonalcoholic fatty liver disease. Nat Genet 2008;40(12):1461-1465

16 Kozlitina J, Smagris E, Stender S, et al. Exome-wide association study identifies a TM6SF2 variant that confers susceptibility to nonalcoholic fatty liver disease. Nat Genet 2014;46(04):352-356

17 Mancina RM, Dongiovanni P, Petta S, et al. The MBOAT7-TMC4 variant rs641738 increases risk of nonalcoholic fatty liver disease in individuals of European descent. Gastroenterology 2016; 150(05):1219-1230.e6

18 Schwimmer JB, McGreal N, Deutsch R, Finegold MJ, Lavine JE. Influence of gender, race, and ethnicity on suspected fatty liver in obese adolescents. Pediatrics 2005;115(05):e561-e565

19 Dongiovanni P, Romeo S, Valenti L. Genetic factors in the pathogenesis of nonalcoholic fatty liver and steatohepatitis. BioMed Res Int 2015;2015:460190

20 Dongiovanni P, Donati B, Fares R, et al. PNPLA3 I148M polymorphism and progressive liver disease. World J Gastroenterol 2013;19(41):6969-6978

21 Dongiovanni P, Petta S, Maglio C, et al. Transmembrane 6 superfamily member 2 gene variant disentangles nonalcoholic steatohepatitis from cardiovascular disease. Hepatology 2015;61 (02):506-514

22 Santoro N, Zhang CK, Zhao H, et al. Variant in the glucokinase regulatory protein $(\mathrm{GCKR})$ gene is associated with fatty liver in obese children and adolescents. Hepatology 2012;55(03): 781-789
23 Suomela E, Oikonen M, Pitkänen N, et al. Childhood predictors of adult fatty liver. The Cardiovascular Risk in Young Finns Study. J Hepatol 2016;65(04):784-790

24 Sun C, Fan JG, Qiao L. Potential epigenetic mechanism in nonalcoholic Fatty liver disease. Int J Mol Sci 2015;16(03): 5161-5179

25 Nobili V, Marcellini M, Marchesini G, et al. Intrauterine growth retardation, insulin resistance, and nonalcoholic fatty liver disease in children. Diabetes Care 2007;30(10):2638-2640

26 Kleiner DE, Brunt EM, Van Natta M, et al; Nonalcoholic Steatohepatitis Clinical Research Network. Design and validation of a histological scoring system for nonalcoholic fatty liver disease. Hepatology 2005;41(06):1313-1321

27 Dongiovanni P, Fracanzani AL, Fargion S, Valenti L. Iron in fatty liver and in the metabolic syndrome: a promising therapeutic target. J Hepatol 2011;55(04):920-932

28 Singh S, Allen AM, Wang Z, Prokop LJ, Murad MH, Loomba R. Fibrosis progression in nonalcoholic fatty liver vs nonalcoholic steatohepatitis: a systematic review and meta-analysis of paired-biopsy studies. Clin Gastroenterol Hepatol 2015;13 (04):643-54.e1, 9, quiz e39-e40

29 Angulo P, Kleiner DE, Dam-Larsen S, et al. Liver fibrosis, but no other histologic features, is associated with long-term outcomes of patients with nonalcoholic fatty liver disease. Gastroenterology 2015;149(02):389-97.e10

30 Ekstedt M, Hagström H, Nasr P, et al. Fibrosis stage is the strongest predictor for disease-specific mortality in NAFLD after up to 33 years of follow-up. Hepatology 2015;61(05):1547-1554

31 Dulai PS, Singh S, Patel J, et al. Increased risk of mortality by fibrosis stage in nonalcoholic fatty liver disease: systematic review and meta-analysis. Hepatology 2017;65(05):1557-1565

32 Hagström H, Nasr P, Ekstedt M, et al. Fibrosis stage but not NASH predicts mortality and time to development of severe liver disease in biopsy-proven NAFLD. J Hepatol 2017;67(06): 1265-1273

33 Schwimmer JB, Behling C, Newbury R, et al. Histopathology of pediatric nonalcoholic fatty liver disease. Hepatology 2005;42 (03):641-649

34 Ko JS, Yoon JM, Yang HR, et al. Clinical and histological features of nonalcoholic fatty liver disease in children. Dig Dis Sci 2009;54 (10):2225-2230

35 Takahashi Y, Inui A, Fujisawa T, Takikawa H, Fukusato T. Histopathological characteristics of non-alcoholic fatty liver disease in children: comparison with adult cases. Hepatol Res 2011;41 (11):1066-1074

36 Skoien R, Richardson MM, Jonsson JR, et al. Heterogeneity of fibrosis patterns in non-alcoholic fatty liver disease supports the presence of multiple fibrogenic pathways. Liver Int 2013;33(04): 624-632

37 Brunt EM, Kleiner DE, Wilson LA, et al; NASH Clinical Research NetworkA list of members of the Nonalcoholic Steatohepatitis Clinical Research Network can be found in the Appendix. Portal chronic inflammation in nonalcoholic fatty liver disease (NAFLD): a histologic marker of advanced NAFLD-Clinicopathologic correlations from the nonalcoholic steatohepatitis clinical research network. Hepatology 2009;49(03):809-820

38 Mann JP, De Vito R, Mosca A, et al. Portal inflammation is independently associated with fibrosis and metabolic syndrome in pediatric nonalcoholic fatty liver disease. Hepatology 2016;63 (03):745-753

39 Scorletti E, Calder PC, Byrne CD. Non-alcoholic fatty liver disease and cardiovascular risk: metabolic aspects and novel treatments. Endocrine 2011;40(03):332-343

40 Byrne CD. Ectopic fat, insulin resistance and non-alcoholic fatty liver disease. Proc Nutr Soc 2013;72(04):412-419

41 Angulo P, Hui JM, Marchesini G, et al. The NAFLD fibrosis score: a noninvasive system that identifies liver fibrosis in patients with NAFLD. Hepatology 2007;45(04):846-854 
42 Byrne CD, Olufadi R, Bruce KD, Cagampang FR, Ahmed MH. Metabolic disturbances in non-alcoholic fatty liver disease. Clin Sci (Lond) 2009;116(07):539-564

43 Kong L, Lu Y, Zhang S, Nan Y, Qiao L. Role of nutrition, gene polymorphism, and gut microbiota in non-alcoholic fatty liver disease. Discov Med 2017;24(131):95-106

44 Modi N, Murgasova D, Ruager-Martin R, et al. The influence of maternal body mass index on infant adiposity and hepatic lipid content. Pediatr Res 2011;70(03):287-291

45 Brumbaugh DE, Tearse P, Cree-Green M, et al. Intrahepatic fat is increased in the neonatal offspring of obese women with gestational diabetes. J Pediatr 2013;162(05):930-6.e1

46 Brumbaugh DE, Friedman JE. Developmental origins of nonalcoholic fatty liver disease. Pediatr Res 2014;75(1-2):140-147

47 Holland ML, Lowe R, Caton PW, et al. Early-life nutrition modulates the epigenetic state of specific rDNA genetic variants in mice. Science 2016;353(6298):495-498

48 Tarry-Adkins JL, Fernandez-Twinn DS, Hargreaves IP, et al. Coenzyme Q10 prevents hepatic fibrosis, inflammation, and oxidative stress in a male rat model of poor maternal nutrition and accelerated postnatal growth. Am J Clin Nutr 2016;103(02): 579-588

49 Alfaradhi MZ, Kusinski LC, Fernandez-Twinn DS, et al. Maternal obesity in pregnancy developmentally programs adipose tissue inflammation in young, lean male mice offspring. Endocrinology 2016;157(11):4246-4256

50 Fernandez-Twinn DS, Gascoin G, Musial B, et al. Exercise rescues obese mothers' insulin sensitivity, placental hypoxia and male offspring insulin sensitivity. Sci Rep 2017;7:44650

51 Sutton EF, Gilmore LA, Dunger DB, et al. Developmental programming: state-of-the-science and future directions-Summary from a Pennington Biomedical symposium. Obesity (Silver Spring) 2016;24(05):1018-1026

52 Wesolowski SR, Kasmi KC, Jonscher KR, Friedman JE. Developmental origins of NAFLD: a womb with a clue. Nat Rev Gastroenterol Hepatol 2017;14(02):81-96

53 Mouralidarane A, Soeda J, Visconti-Pugmire C, et al. Maternal obesity programs offspring nonalcoholic fatty liver disease by innate immune dysfunction in mice. Hepatology 2013;58(01): 128-138

54 Li M, Reynolds CM, Segovia SA, Gray C, Vickers MH. Developmental programming of nonalcoholic fatty liver disease: the effect of early life nutrition on susceptibility and disease severity in later life. BioMed Res Int 2015;2015:437107

55 Herrera E, Amusquivar E. Lipid metabolism in the fetus and the newborn. Diabetes Metab Res Rev 2000;16(03):202-210

56 Bruce KD, Cagampang FR, Argenton M, et al. Maternal high-fat feeding primes steatohepatitis in adult mice offspring, involving mitochondrial dysfunction and altered lipogenesis gene expression. Hepatology 2009;50(06):1796-1808

57 Hales CN, Barker DJP. The thrifty phenotype hypothesis. Br Med Bull 2001;60:5-20

58 Bugianesi E, Bizzarri C, Rosso C, et al. Low birthweight increases the likelihood of severe steatosis in pediatric non-alcoholic fatty liver disease. Am J Gastroenterol 2017;112(08):1277-1286

59 Clària J, González-Périz A, López-Vicario C, Rius B, Titos E. New insights into the role of macrophages in adipose tissue inflammation and fatty liver disease: modulation by endogenous omega-3 fatty Acid-derived lipid mediators. Front Immunol 2011;2:49

60 Rius B, López-Vicario C, González-Périz A, et al. Resolution of inflammation in obesity-induced liver disease. Front Immunol 2012;3:257

61 Moran A, Jacobs DR Jr, Steinberger J, et al. Insulin resistance during puberty: results from clamp studies in 357 children. Diabetes 1999;48(10):2039-2044

62 Moran A, Jacobs DR Jr, Steinberger J, et al. Changes in insulin resistance and cardiovascular risk during adolescence: establish- ment of differential risk in males and females. Circulation 2008; 117(18):2361-2368

63 Vos MB, Kimmons JE, Gillespie C, Welsh J, Blanck HM. Dietary fructose consumption among US children and adults: the Third National Health and Nutrition Examination Survey. Medscape J Med 2008;10(07):160

64 Softic S, Gupta MK, Wang GX, et al. Divergent effects of glucose and fructose on hepatic lipogenesis and insulin signaling. J Clin Invest 2017;127(11):4059-4074

65 Geisler CE, Renquist BJ. Hepatic lipid accumulation: cause and consequence of dysregulated glucoregulatory hormones. J Endocrinol 2017;234(01):R1-R21

66 Solinas G, Borén J, Dulloo AG. De novo lipogenesis in metabolic homeostasis: more friend than foe? Mol Metab 2015;4(05): 367-377

67 Bode JC, Zelder O, Rumpelt HJ, Wittkamp U. Depletion of liver adenosine phosphates and metabolic effects of intravenous infusion of fructose or sorbitol in man and in the rat. Eur J Clin Invest 1973;3(05):436-441

68 Crescenzo R, Bianco F, Falcone I, Coppola P, Liverini G, Iossa S. Increased hepatic de novo lipogenesis and mitochondrial efficiency in a model of obesity induced by diets rich in fructose. Eur J Nutr 2013;52(02):537-545

69 Softic S, Cohen DE, Kahn CR. Role of dietary fructose and hepatic de novo lipogenesis in fatty liver disease. Dig Dis Sci 2016;61 (05):1282-1293

70 Diggle CP, Shires M, Leitch D, et al. Ketohexokinase: expression and localization of the principal fructose-metabolizing enzyme. J Histochem Cytochem 2009;57(08):763-774

71 Petrie JL, Patman GL, Sinha I, Alexander TD, Reeves HL, Agius L. The rate of production of uric acid by hepatocytes is a sensitive index of compromised cell ATP homeostasis. Am J Physiol Endocrinol Metab 2013;305(10):E1255-E1265

72 Serdula MK, Ivery D, Coates RJ, Freedman DS, Williamson DF, Byers T. Do obese children become obese adults? A review of the literature. Prev Med 1993;22(02):167-177

73 Feldstein AE, Charatcharoenwitthaya P, Treeprasertsuk S, Benson JT, Enders FB, Angulo P. The natural history of non-alcoholic fatty liver disease in children: a follow-up study for up to 20 years. Gut 2009;58(11):1538-1544

74 Molleston JP, White F, Teckman J, Fitzgerald JF. Obese children with steatohepatitis can develop cirrhosis in childhood. Am J Gastroenterol 2002;97(09):2460-2462

75 Vajro P, Lenta S, Socha P, et al. Diagnosis of nonalcoholic fatty liver disease in children and adolescents: position paper of the ESPGHAN Hepatology Committee. J Pediatr Gastroenterol Nutr 2012;54(05):700-713

76 Adams LA, Feldstein A, Lindor KD, Angulo P. Nonalcoholic fatty liver disease among patients with hypothalamic and pituitary dysfunction. Hepatology 2004;39(04):909-914

77 Unalp-Arida A, Ruhl CE. Liver fibrosis scores predict liver disease mortality in the United States population. Hepatology 2017;66 (01):84-95

78 Nobili V, Alisi A, Vania A, Tiribelli C, Pietrobattista A, Bedogni G. The pediatric NAFLD fibrosis index: a predictor of liver fibrosis in children with non-alcoholic fatty liver disease. BMC Med 2009; $7: 21$

79 Lavine JE, Schwimmer JB, Van Natta ML, et al; Nonalcoholic Steatohepatitis Clinical Research Network. Effect of vitamin E or metformin for treatment of nonalcoholic fatty liver disease in children and adolescents: the TONIC randomized controlled trial. JAMA 2011;305(16):1659-1668

80 Nobili V, Manco M, Devito R, et al. Lifestyle intervention and antioxidant therapy in children with nonalcoholic fatty liver disease: a randomized, controlled trial. Hepatology 2008;48 (01):119-128

81 Barritt AS IV, Gitlin N, Klein S, et al. Design and rationale for a real-world observational cohort of patients with nonalcoholic 
fatty liver disease: the TARGET-NASH study. Contemp Clin Trials 2017;61:33-38

82 Mann J, et al. European Paediatric NAFLD Registry (EU-PNAFLD). 2017. Project ID: 174534 (UK)

83 Feldman A, Eder SK, Felder TK, et al. Clinical and metabolic characterization of lean Caucasian subjects with non-alcoholic fatty liver. Am J Gastroenterol 2017;112(01):102-110

84 Parker VE, Semple RK. Genetics in endocrinology: genetic forms of severe insulin resistance: what endocrinologists should know. Eur J Endocrinol 2013;169(04):R71-R80

85 Mak CM, Lam CW, Tam S. Diagnostic accuracy of serum ceruloplasmin in Wilson disease: determination of sensitivity and specificity by ROC curve analysis among ATP7B-genotyped subjects. Clin Chem 2008;54(08):1356-1362

86 European Association for Study of Liver. EASL Clinical Practice Guidelines: Wilson's disease. J Hepatol 2012;56(03):671-685

87 Burton BK, Deegan PB, Enns GM, et al. Clinical features of lysosomal acid lipase deficiency. J Pediatr Gastroenterol Nutr 2015;61(06):619-625

88 Burton BK, Balwani M, Feillet F, et al. A phase 3 trial of sebelipase alfa in lysosomal acid lipase deficiency. N Engl J Med 2015;373 (11):1010-1020

89 Selvakumar PK, Kabbany MN, Lopez R, et al. Reduced lysosomal acid lipase activity - a potential role in the pathogenesis of non alcoholic fatty liver disease in pediatric patients. Dig Liver Dis 2016;48(08):909-913

90 Molleston JP, Schwimmer JB, Yates KP, et al; NASH Clinical Research Network. Histological abnormalities in children with nonalcoholic fatty liver disease and normal or mildly elevated alanine aminotransferase levels. J Pediatr 2014;164(04): 707-713.e3

91 Burgert TS, Taksali SE, Dziura J, et al. Alanine aminotransferase levels and fatty liver in childhood obesity: associations with insulin resistance, adiponectin, and visceral fat. J Clin Endocrinol Metab 2006;91(11):4287-4294

92 Chalasani N, Younossi Z, Lavine JE, et al. The diagnosis and management of nonalcoholic fatty liver disease: practice guidance from the American Association for the Study of Liver Diseases. Hepatology 2018;67(01):328-357

93 Vajro P, Mandato C, Franzese A, et al. Vitamin E treatment in pediatric obesity-related liver disease: a randomized study. J Pediatr Gastroenterol Nutr 2004;38(01):48-55

94 Boyraz M, Pirgon Ö, Dündar B, Çekmez F, Hatipoğlu N. Long-term treatment with n-3 polyunsaturated fatty acids as a monotherapy in children with nonalcoholic fatty liver disease. J Clin Res Pediatr Endocrinol 2015;7(02):121-127

95 Gibson PS, Lang S, Dhawan A, et al. Systematic review: nutrition and physical activity in the management of paediatric nonalcoholic fatty liver disease. J Pediatr Gastroenterol Nutr 2017;65 (02):141-149

96 Ramon-Krauel M, Salsberg SL, Ebbeling CB, et al. A low-glycemicload versus low-fat diet in the treatment of fatty liver in obese children. Child Obes 2013;9(03):252-260

97 Vos MB, Weber MB, Welsh J, et al. Fructose and oxidized lowdensity lipoprotein in pediatric nonalcoholic fatty liver disease: a pilot study. Arch Pediatr Adolesc Med 2009;163(07):674-675

98 Goss A. A Carbohydrate-Restricted Diet to Reverse Fatty Liver in Adolescents with Obesity. Birmingham, AL: University of Alabama at Birmingham; 2017

99 O'Connor EA, Evans CV, Burda BU, Walsh ES, Eder M, Lozano P. Screening for obesity and intervention for weight management in children and adolescents: evidence report and systematic review for the US Preventive Services Task Force. JAMA 2017;317 (23):2427-2444

100 Schwimmer JB. Clinical advances in pediatric nonalcoholic fatty liver disease. Hepatology 2016;63(05):1718-1725

101 Akcam M, Boyaci A, Pirgon O, Kaya S, Uysal S, Dundar BN. Therapeutic effect of metformin and vitamin E versus prescrip- tive diet in obese adolescents with fatty liver. Int J Vitam Nutr Res 2011;81(06):398-406

102 Zöhrer E, Alisi A, Jahnel J, et al. Efficacy of docosahexaenoic acidcholine-vitamin E in paediatric NASH: a randomized controlled clinical trial. Appl Physiol Nutr Metab 2017;42(09):948-954

103 Wang CL, Liang L, Fu JF, et al. Effect of lifestyle intervention on non-alcoholic fatty liver disease in Chinese obese children. World J Gastroenterol 2008;14(10):1598-1602

104 Schwimmer JB, Lavine JE, Wilson LA, et al; NASH CRN. In children with nonalcoholic fatty liver disease, cysteamine bitartrate delayed release improves liver enzymes but does not reduce disease activity scores. Gastroenterology 2016;151(06):1141-1154.e9

105 Shiasi Arani K, Taghavi Ardakani A, Moazami Goudarzi R, et al. Effect of vitamin $\mathrm{E}$ and metformin on fatty liver disease in obese children- randomized clinical trial. Iran J Public Health 2014;43 (10):1417-1423

106 Nadeau KJ, Ehlers LB, Zeitler PS, Love-Osborne K. Treatment of non-alcoholic fatty liver disease with metformin versus lifestyle intervention in insulin-resistant adolescents. Pediatr Diabetes 2009;10(01):5-13

107 Pastor-Villaescusa B, Cañete MD, Caballero-Villarraso J, et al. Metformin for obesity in prepubertal and pubertal children: a randomized controlled trial. Pediatrics 2017;140(01):e20164285

108 Nobili V, Alisi A, Della Corte C, et al. Docosahexaenoic acid for the treatment of fatty liver: randomised controlled trial in children. Nutr Metab Cardiovasc Dis 2013;23(11):1066-1070

109 Pacifico L, Bonci E, Di Martino M, et al. A double-blind, placebocontrolled randomized trial to evaluate the efficacy of docosahexaenoic acid supplementation on hepatic fat and associated cardiovascular risk factors in overweight children with nonalcoholic fatty liver disease. Nutr Metab Cardiovasc Dis 2015;25(08): 734-741

110 Alisi A, Bedogni G, Baviera G, et al. Randomised clinical trial: the beneficial effects of VSL\#3 in obese children with non-alcoholic steatohepatitis. Aliment Pharmacol Ther 2014;39(11):1276-1285

111 Famouri F, Shariat Z, Hashemipour M, Keikha M, Kelishadi R. Effects of probiotics on nonalcoholic fatty liver disease in obese children and adolescents. J Pediatr Gastroenterol Nutr 2017;64 (03):413-417

112 Henao-Mejia J, Elinav E, Jin C, et al. Inflammasome-mediated dysbiosis regulates progression of NAFLD and obesity. Nature 2012;482(7384):179-185

113 Schnabl B, Brenner DA. Interactions between the intestinal microbiome and liver diseases. Gastroenterology 2014;146 (06):1513-1524

114 Leung DH, Yimlamai D. The intestinal microbiome and paediatric liver disease. Lancet Gastroenterol Hepatol 2017;2(06):446-455

115 Olson ML, Maalouf NM, Oden JD, White PC, Hutchison MR. Vitamin D deficiency in obese children and its relationship to glucose homeostasis. J Clin Endocrinol Metab 2012;97(01): 279-285

116 Eliades M, Spyrou E, Agrawal N, et al. Meta-analysis: vitamin D and non-alcoholic fatty liver disease. Aliment Pharmacol Ther 2013;38(03):246-254

117 Della Corte C, Carpino G, De Vito R, et al. Docosahexanoic acid plus vitamin $D$ treatment improves features of NAFLD in children with serum vitamin $\mathrm{D}$ deficiency: results from a single centre trial. PLoS One 2016;11(12):e0168216

118 Manco M, Mosca A, De Peppo F, et al. The benefit of sleeve gastrectomy in obese adolescents on nonalcoholic steatohepatitis and hepatic fibrosis. J Pediatr 2017;180:31-37.e2

119 Nobili V, Vajro P, Dezsofi A, et al. Indications and limitations of bariatric intervention in severely obese children and adolescents with and without nonalcoholic steatohepatitis: ESPGHAN Hepatology Committee Position Statement. J Pediatr Gastroenterol Nutr 2015;60(04):550-561

120 Chavez-Tapia NC, Tellez-Avila FI, Barrientos-Gutierrez T, Mendez-Sanchez N, Lizardi-Cervera J, Uribe M. Bariatric surgery for 
non-alcoholic steatohepatitis in obese patients. Cochrane Database Syst Rev 2010;CD007340(01):CD007340

121 Sjöström L, Narbro K, Sjöström CD, et al; Swedish Obese Subjects Study. Effects of bariatric surgery on mortality in Swedish obese subjects. N Engl J Med 2007;357(08):741-752

122 Zeller MH, Pendery EC, Reiter-Purtill J, et al. From adolescence to young adulthood: trajectories of psychosocial health following Rouxen-Y gastric bypass. Surg Obes Relat Dis 2017;13(07):1196-1203

123 Ayonrinde OT, Oddy WH, Adams LA, et al. Infant nutrition and maternal obesity influence the risk of non-alcoholic fatty liver disease in adolescents. J Hepatol 2017;67(03):568-576

124 Nobili V, Bedogni G, Alisi A, et al. A protective effect of breastfeeding on the progression of non-alcoholic fatty liver disease. Arch Dis Child 2009;94(10):801-805

125 Breij LM, Kerkhof GF, Hokken-Koelega ACS. Accelerated infant weight gain and risk for nonalcoholic fatty liver disease in early adulthood. J Clin Endocrinol Metab 2014;99(04):1189-1195
126 Sandboge S, Perälä MM, Salonen MK, et al. Early growth and nonalcoholic fatty liver disease in adulthood-the NAFLD liver fat score and equation applied on the Helsinki Birth Cohort Study. Ann Med 2013;45(5-6):430-437

127 Fraser A, Ebrahim S, Smith GD, Lawlor DA. The associations between birthweight and adult markers of liver damage and function. Paediatr Perinat Epidemiol 2008;22(01):12-21

128 Jin R, Welsh JA, Le NA, et al. Dietary fructose reduction improves markers of cardiovascular disease risk in Hispanic-American adolescents with NAFLD. Nutrients 2014;6(08):3187-3201

129 Janczyk W, Lebensztejn D, Wierzbicka-Rucińska A, et al. Omega3 fatty acids therapy in children with nonalcoholic fatty liver disease: a randomized controlled trial. J Pediatr 2015;166(06): 1358-63.e1, 3

130 Vajro P, Mandato C, Licenziati MR, et al. Effects of Lactobacillus rhamnosus strain GG in pediatric obesity-related liver disease. J Pediatr Gastroenterol Nutr 2011;52(06):740-743 